\title{
Telencephalic Lesions and Behavior in the Teleost Macropodus opercularis (L.): Effects of Telencephalon and Olfactory Bulb Ablation on Spawning and Foamnest Building ${ }^{1}$
}

\author{
Patricia Schwagmeyer, Roger E. Davis, and Jeffrey Kassel
}

The Mental Health Research Institute and The Neuroscience Laboratory, The University of Michigan, Ann Arbor, Michigan 48109

\begin{abstract}
Behavior during 5-day spawning trials of intact, sham-operated, olfactory bulb-ablated, and telencephalon-ablated male paradise fish, Macropodus opercularis, was investigated. Spawning and nestbuilding were eliminated in the telencephalon-ablated males and unimpaired in the sham-operated and olfactory bulb-ablated individuals. Telencephalon ablation resulted in decreased frequencies of frontal display, attack, and bubble blowing and differences in the probability of certain behavior sequences. The data indicate that the block of reproduction is a result of interference with nonolfactory functions of the telencephalon.
\end{abstract}

Telencephalon ablation was previously reported to block spawning and foamnest building in male Macropodus opercularis (Davis et al., 1976). Lesioned males paired individually with intact females showed decreased frequencies of social behaviors at the start of the spawning trial, and during the trial they were subordinate to the female. The present study was undertaken to investigate in greater detail the effects of telencephalon ablation on reproductive behavior.

The telencephalon ablation resulted in the destruction of the olfactory system. Olfactory stimuli strongly influence social behavior in fishes (Atema et al., 1969), including Macropodus (Rossi, 1969; Davis and Pilotte, 1975). The loss of olfaction rather than of nonolfactory functions of the telencephalon may be responsible for the block of reproduction. To examine this possibility we investigated the effects of olfactory bulb ablation on sociosexual behavior.

\section{METHODS}

Forty adult male Macropodus opercularis (L.), mean body length of 4.8 $\mathrm{cm}$ and mean body weight of $3.4 \mathrm{~g}$, were distributed among the following four experimental groups: intact, sham-operated (sham), olfactory bulb-

1 We thank Gertrude Calloway, Linda Walls, and Robin Reynolds for their assistance. 
ablated (ob-x), and telencephalon-ablated (tele-x). Prior to the experiment, fish were kept in bisexual groups in large tanks. The previous sexual experience of the males was unknown. Commercial holding tanks and crowded storage tanks in the laboratory generally are incompatible with reproduction in Macropodus. The fish were fed Tetramin Staple Conditioning food or frozen brine shrimp daily. Water temperature was maintained at 24 to $26^{\circ} \mathrm{C}$. The daily light period consisted of 14:10-hr L:D cycle of "daylight" fluorescent light. Several days preceding surgery, males were transferred from the stock tanks to individual isolation tanks (Davis et al., 1974). The male could see other males in the adjacent tanks.

Craniotomy and extirpation of the telencephalon, including the olfactory bulbs, were carried out as previously described (Davis et al., 1976). In the ob-x fish, the olfactory bulbs were transected beneath the rostral end of the telencephalon and then aspirated. The sham operation consisted of the craniotomy and removal of some of the lipoid tissue over the telencephalon.

Sham, ob-x, and tele-x males were individually administered two 5-day spawning trials starting 4 and 18 days following surgery. The 10 intact males received the first trial only. In a trial, the male was placed in a 38-liter tank with an intact adult female which had been placed in the tank the previous day. Water temperatures were 26 to $28^{\circ} \mathrm{C}$. Males which spawned prior to Day 5 were returned to the isolation tank to await a second trial or were sacrificed preparatory to brain histology. In a few instances, the first trial was halted because the tele- $x$ male was injured by the female. The injured male was isolated for up to a week to permit its recovery, then paired with a female in another 5-day trial. Only data from complete trials were included in the results. The tele- $x$ group was reduced to eight males when two died following attacks by the female. One sham died of unknown causes during a spawning trial.

The male's behavior was observed twice daily for $10 \mathrm{~min}$ between 0800 and $1000 \mathrm{hr}$ and between 1300 and $1500 \mathrm{hr}$ (EDT). The following units of behavior were recorded: approach, lateral and frontal display, quiver, chase, attack, bubble, curve and clasp, and pause. Approach, lateral and frontal display, quiver, chase, and attack have previously been described (Davis and Kassel, 1975). Bubble consisted of the release of bubbles of air from the mouth. Bubble did not necessarily result in the construction of a foamnest. Curve was denoted as a lateral C-shaped bend of the male's body. In clasp, the male's head and caudal fin wrapped around the anterior half of the femaie's body (Hall, 1968). Pruse signiffed the nonoccurrence of the other units for period of $10 \mathrm{sec}$ or longer. The frequency and the temporal sequence of the behaviors were registered with a manual keyboard and a PDP-8F digital computer. The approximate surface area of a foamnest, the relative conesiveness of a nest, and the presence of nests built by the female were recorded on prepared charts in each observation period. 
Upon completion of the spawning trials the males were killed in ice water. The head was removed, fixed in formalin, decalcified, and embedded in paraffin. Coronal, $8-\mu \mathrm{m}$ sections were cut and stained with hematoxylin and eosin. The extent of the ablations was determined by examination of serial sections of intact and lesioned brains, using Peter and Gill's (1975) atlas as a guide to teleost brain anatomy.

\section{RESULTS}

None of the tele-x males spawned during the 5-day trials, whereas nine of the ten intact males spawned. Eight of the nine shams and the eight ob-x males spawned in one or both trials (Table I). All of the intact, sham, and ob-x males, but no tele-x male, built a foamnest. The nest surface area did not differ between the groups. Individual nests ranged from 6 to $55 \mathrm{~cm}^{2}$. The lesioned males showed no impairment of swimming or feeding.

The mean frequencies of the behaviors did not differ significantly between trials 1 and 2 for the sham, ob-x, and tele-x males (Student's $t$ test). The data for the two trials were thus combined to obtain group mean values which could be contrasted with the data for the intact males. A one-way analysis of variance (ANOVA) for each behavior variable revealed significant differences between the groups in the frequencies of frontal display, attack, and bubble. Table 1 shows that these behaviors were decreased in the tele- $x$ males. The frequencies of frontal display and bubble were not significantly different (Scheffé $t$ test) in ob-x, sham, and

TABLE 1

Mean Frequency of Behaviors During the 10-min Observation Periods in the Spawning Trial and the Spawning Frequency for Intact,

Sham-Operated and Brain-Lesioned Males

Behavior

Lesion

\begin{tabular}{|c|c|c|c|c|c|c|}
\hline & & & & & & \\
\hline & $\begin{array}{c}\text { Intact } \\
N=10\end{array}$ & $\begin{array}{l}\text { Sham } \\
N=9\end{array}$ & $\begin{array}{c}\text { Ob-x } \\
N=8\end{array}$ & $\begin{array}{c}\text { Tele-x } \\
N=8\end{array}$ & $F$ & $P$ \\
\hline Approach & 8.2 & 6.8 & 8.0 & 6.1 & $<1$ & ns \\
\hline Lateral display & 9.5 & 10.8 & 7.7 & 10.1 & $<1$ & ns \\
\hline Frontal display & 10.5 & 10.4 & 7.4 & 4.7 & 3.9 & $<0.05$ \\
\hline Chase & 7.3 & 5.8 & 4.9 & 2.4 & 2.4 & ns \\
\hline Atrack & 3.6 & 0.9 & 3.3 & 0.5 & 5.4 & $<0.005$ \\
\hline Quiver & 0.5 & 0.6 & 0.5 & 0.8 & $<1$ & $\mathrm{~ns}$ \\
\hline Bubble & 3.2 & 3.6 & 2.8 & 0.8 & 7.5 & $<0.001$ \\
\hline Curve & 0.8 & 0.4 & 0.2 & $0.0:$ & 13 & ns \\
\hline Ciasp & 3.98 & $0.1 \mathrm{i}$ & 0.04 & 0.0 & $\therefore .1$ & $\mathbb{n} s$ \\
\hline Trejos & 21.3 & 10.6 & 12.3 & $10 . \hat{5}$ & $\therefore$ & ns \\
\hline $\begin{array}{l}\text { Wumber spawned } \\
\text { Humber spawred }\end{array}$ & 9 & 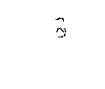 & 8 & 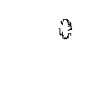 & & \\
\hline Dpontuntiss & $\$ / 10$ & 1318 & 2210 & Oh & & \\
\hline
\end{tabular}


intact males. The tele- $x$ and sham groups showed equivalent low frequencies of attack; thus the possible influence of the ablation on this behavior was unclear. One tele-x male was seen to bubble late on Day 3 of the trial. The female had previously constructed a diffuse nest in the area and was maintaining it. The next morning the male performed a curve when the female nudged its flank and bubbled during the afternoon observation. No further bubbling or sexual behavior by the male was observed in the trial.

Two-unit sequence tables were constructed for sham and tele-x males (Table 2). The frequencies of the various two-unit sequences were converted to probabilities by division of cell frequencies by row totals. Sequences for which transitional probability was less than or equal to 0.10 were arbitrarily designated as random; sequences in which the total frequency of the "preceding unit" was less than 85 were also excluded. The tele-x males paused relatively more frequently following frontal display, attack, chase, lateral display, and approach than did the shams. Approach was less likely to be followed by frontal and lateral display and chase in tele- $x$ than in sham. The sequence of chase-attack was less probable in the tele- $x$ males. In addition, the occurrence of chase-frontal display, frontal-chase, and lateral-frontal display were less likely in tele- $x$ males. Successive lateral displays were more frequent in the tele- $x$ than in the sham males. Our strong impression was that the displays by the tele- $x$ males were primarily elicited by an approach or display by the

TABLE 2

Comparison of Probabilities of Selected Two-Unit Sequences Shown by Sham-Operated ${ }^{a}$ and the Tele-x Males during the Spawning Trial ${ }^{b}$

\begin{tabular}{lcccccr}
\hline Preceding unit & \multicolumn{5}{c}{ Following unit } \\
\cline { 2 - 7 } & $\begin{array}{l}\text { Lateral } \\
\text { display }\end{array}$ & $\begin{array}{c}\text { Frontal } \\
\text { display }\end{array}$ & Chase & Attack & Pause & $\begin{array}{r}\text { Unit } \\
\text { total }\end{array}$ \\
\hline Approach & $0.14^{*}$ & $0.27^{*}$ & $0.12^{*}$ & & $0.41^{*}$ & 1072 \\
& $(0.19)$ & $(0.36)$ & $(0.17)$ & & $(0.21)$ & 684 \\
Lateral & $0.27^{*}$ & $-^{*}$ & & & $0.49^{*}$ & 1767 \\
$\quad$ display & $(0.18)$ & $(0.17)$ & & & $(0.36)$ & 963 \\
Frontal & 0.36 & & $0.17^{*}$ & & $0.29^{*}$ & 819 \\
$\quad$ display & $(0.35)$ & & $(0.28)$ & & $(0.12)$ & 929 \\
Chase & & $0.18^{*}$ & & $\mathbf{Z}^{*}$ & $0.57^{*}$ & 421 \\
& & $(0.47)$ & & $(0.12)$ & $(0.22)$ & 538 \\
Attack & & & 0.18 & & $0.57^{*}$ & 87 \\
& & & $(0.30)$ & & $(0.30)$ & 105 \\
Pause & $0.38^{*}$ & & & & & 1884 \\
& $(0.16)$ & & & & & \\
\hline
\end{tabular}

a Values in parentheses.

${ }^{b}$ Sequence probabilities equal to or less than 0.10 were omitted. Sequences based on less than a total of 85 units were omitted.

$\chi^{2}$ test, $P<0.05$. 
female. Owing to the limited data, analysis of the dyadic interactions is not possible.

The frequency of certain behaviors changed during the spawning trial. Curve, clasp, and bubble increased prior to spawning. To determine whether changes in the frequency of the other behaviors within a trial were different between sham and tele-x males, we contrasted the data from the first and last 10-min observation periods of trial 1 (Table 3). A two-factor mixed design ANOVA showed a significant time effect on lateral display $(F=10.9, d f 1 / 15, P<0.005)$, frontal display $(F=7.5, P<0.025)$, quiver $(F=7.7, P<0.025)$, and attack $(F=7.3, P<0.025)$. The lesion effect was significant for frontal display $(F=6.9, P<0.025)$. A significant time by lesion interaction was indicated only in the frequency of approach $(F$ $=5.06, P<0.025$ ). Approach increased in frequency for the tele- $\mathrm{x}$ males and decreased for the shams.

In seven of the eight tele-x males, both telencephalic hemispheres and olfactory bulbs were removed at the anterior commissure or the rostral end of the telencephalic peduncle (Fig. 1). The lesion variably invaded the anterior portion of the preoptic nucleus, but the nucleus preopticus pars magnocellularis was intact. The eighth male retained a small portion of the caudal subpallium and of area dorsalis telencephali pars lateralis on the right side. Complete bilateral bulbectomy was achieved in seven of the eight ob-x males; in one, the left bulb was ablated and the right bulb was extensively damaged but not fully separated from the olfactory nerve. There was no within-group behavioral variation corresponding to the variation in extent of the ob-x and tele-x lesions.

\section{DISCUSSION}

The observed impairment of reproductive behavior following telencephalon ablation in Macropodus resembles the results obtained in Betta, Hemichromis, and Gasterosteus (Noble and Borne, 1941; Segaar,

TABLE 3

Mean Frequency of Selected Behaviors in the First and Last 10-min Observation Period of the First Spawning Trial for

Sham-Operated and Tele-x Males

\begin{tabular}{lrrrrrr}
\hline \multirow{2}{*}{ Behavior } & \multicolumn{2}{c}{$\begin{array}{c}\text { Sham males } \\
\text { 10-min period }\end{array}$} & & \multicolumn{2}{c}{$\begin{array}{c}\text { Tele-x males } \\
10 \text {-min period }\end{array}$} \\
\cline { 2 - 3 } \cline { 5 - 6 } & First & Last & & & First & Last \\
\hline Approach & 8.1 & 5.6 & & 2.6 & 7.5 \\
Lateral display & 22.9 & 5.2 & & 22.4 & 7.8 \\
Frontal display & 18.9 & 6.1 & & 6.0 & 2.4 \\
Chase & 5.1 & 4.4 & & 0.5 & 4.2 \\
Attack & 0.4 & 1.1 & & 0.1 & 0.9 \\
Quiver & 3.0 & 0.2 & & 1.1 & 0.0 \\
\hline
\end{tabular}




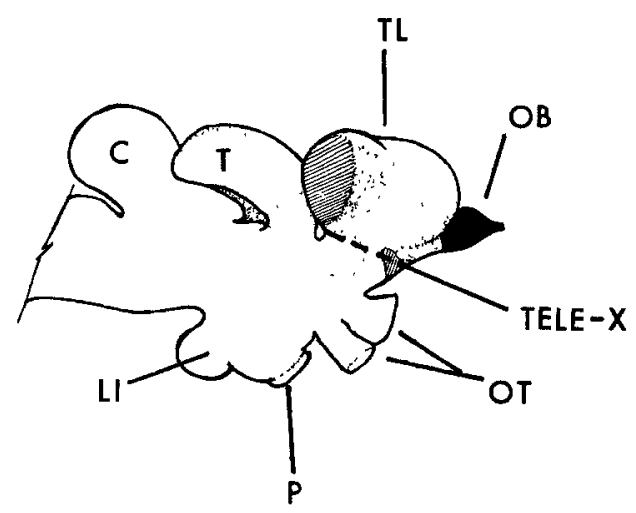

FIG. 1. Medial surface of the paradise fish brain showing the extent of the tele- $x$ and ob-x lesions. Tele-x consisted of bilateral removal of the olfactory bulb and the telencephalon rostral to the dashed line. In ob-x males, the olfactory bulbs were removed as represented by the solid black area. Crosshatched areas: ventral, the anterior commissure; dorsal, zone of pallial coalescence. Abbreviations: C, cerebellum; LI, inferior lobe; OB, olfactory bulb; OT, optic tracts; P, pituitary; T, tectum; TL, telencephalon.

1961, 1965). In Tilapia and Xiphophorus spawning and nestbuilding were disturbed but some fertilization occurred (Aronson, 1948; Kamrin and Aronson, 1954; Overmier and Gross, 1974). The limited, unlocalized bubble behavior and the single curve response which were performed by one tele- $x$ male signify that the telencephalon is not exclusively responsible for the organization of some reproductive behaviors. No bubble, curve, or clasp was seen in tele-x males prior to this experiment. Another individual has since been observed to bubble, however, and to build a disorganized, or scattered, foamnest during a spawning trial 2 weeks following surgery.

The injurious, sometimes lethal female attacks and the decreased frequencies of male frontal display and attack (Table 1) correspond with our previous report that tele-x males were dominated by the intact females (Davis et al., 1976). In both cases, spawning trials were administered within 2 weeks after surgery. Owing to the short postoperative period, the male may not have sufficiently recovered from the trauma of surgery to be able to respond appropriately to the female. The consequent aversive stimulation by the female could result in further inhibition of foamnest building and sexual behavior. Surgical trauma and inhibition owing to aversive stimulation, however, are probably not exclusively responsible for the block of spawning and decreased bubble behavior. Subsequent experiments indicate that the conspicuous male subordinance can be eliminated by increasing the postsurgery recovery period to several weeks (Kassel et al., 1976; Kassel and Davis, in preparation) but that sexual behavior and nestbuilding is nevertheless blocked or severely impaired. 
The loss of olfaction and, more generally, of olfactory bulb function, which follows a complete extirpation of the telencephalon, is not responsible for the decreased frequencies of social behavior and the impairment of spawning and foamnest building. Male and female Macropodus are attracted to fish chemical stimuli (Davis and Pilotte, 1975), and olfaction may and probably does contribute variously to reproductive success in a natural environment. But in a small tank with a single female and without competing males, olfactory bulb-ablated males showed no behavioral impairment. Similar results were obtained in Gasterosteus and Xiphophorus (Segaar, 1961; Kamrin and Aronson, 1954), while catfish social behavior is drastically disrupted following a block of olfactory input (Atema et al., 1969). We conclude that telencephalon ablation disrupts reproduction in Macropodus by removing nonolfactory functions of the telencephalon.

\section{REFERENCES}

Aronson, L. R. (1948). Problems in the behavior and physiology of a species of African mouthbreeding fish. Trans. N.Y. Acad. Sci. 2, 33-42.

Atema, J., Todd, J. H., and Bardach, J. E. (1969). Olfaction and behavioral sophistication in fish. In C. Pfaffman (Ed.), "Olfaction and Taste III," pp. 241-251. New York: Rockefeller University Press.

Davis, R. E., Harris, C., and Shelby, J. (1974). Sex differences in aggressivity and the effects of social isolation in the anabantoid fish, Macropodus opercularis. Behav. Biol. 11, 497-509.

Davis, R. E., and Kassel, J. (1975). The ontogeny of agonistic behavior and the onset of sexual maturation in the paradise fish, Macropodus oeprcularis (Linnaeus). Behav. Biol. 14, 31-39.

Davis, R. E., Kassel, J., and Schwagmeyer, P. (1976). Telencephalic lesions and behavior in the teleost, Macropodus opercularis: Reproduction, startle reaction and operant behavior in the male. Behav. Biol. 18, 165-177.

Davis, R. E., and Pilotte, N. J. (1975). Attraction to conspecific and nonconspecific chemical stimuli in male and female Macropodus opercularis (Teleostei, Anabantoidei). Behav. Biol. 13, 191-196.

Hall, D. D. (1968). A quantitative analysis of courtship and reproductive behavior in the paradise fish, Macropodus opercularis (Linnaeus). Z. Tierpsychol. 25, 834-842.

Kamrin, R. P.. and Aronson, L. R. (1954). The effects of forebrain lesions on mating behavior in the male platyfish, Xiphophorus maculatus. Zoologica (New York) 39, 133-140.

Kassel, J., Davis. R. E., and Schwagmeyer, P. (1976). Telencephalic lesions and behavior in the teleost Macropodus opercularis: Further analysis of reproductive and operant behavior in the male. Behav. Biol. 18, 179-188.

Noble, G. K., and Borne, R. (1941). The effect of forebrain lesions on the sexual and fighting behavior of Betta splendens and other fishes. Anat. Rec. 79, Suppl. 49.

Overmier, J. B., and Gross, D. (1974). Effects of telencephalic ablation upon nest building and avoidance behavior in East African mouthbreeding fish, Tilapia mossambica. Behav. Biol. 12, 211-222.

Peter, R. E., and Gill, V. E. (1975). A stereotaxic atlas and technique for forebrain nuclei of the goldfish, Carassius auratus. J. Comp. Neurol. 159, 69-102. 
Rossi, A. C. (1969). Chemical signals and nest-building in two species of Colisa (Pisces, Anabantidae). Monit. Zool. Ital. 3, 225-237.

Segaar, J. (1961). Telencephalon and behaviour in Gasterosteus aculeatus. Behaviour 18, 256-287.

Segaar, J. (1965). Behavioral aspects of degeneration and regeneration in fish brain: A comparison with higher vertebrates. Progr. Brain Res. 14, 143-231. 